\title{
Diagnostic Disputes Regarding Atypical Melanocytic Lesions can be Solved by Using the Term MELTUMP
}

To the Editor,

Melanocytic tumors of unknown malignant potential (MELTUMPs) are melanocytic lesions that cannot be classified as either benign or malignant tumors. In fact, they have ambiguous characteristics that reflect an unclear biological potential. Several types of melanocytic lesions may be classified as MELTUMPs: atypical Spitz nevi / tumors, dysplastic nevi, pigmented epithelioid melanocytoma, deep penetrating nevi, congenital nevi with atypia, cellular nodules in congenital naevi, borderline melanoma, minimal deviation melanoma, and dermalbased borderline melanocytic tumors. By definition, MELTUMP is a provisional diagnosis and it is necessary to establish parameters that may suggest its most probable clinical behavior. In our experience, the histopathological report should include all the microstaging data for invasive melanoma with the following adjuncts: presence or absence of lymphatic invasion, result of fluorescence in situ hybridization (FISH), histological criteria in according to Cerroni and colleagues (1), and second opinion consultation. Lymphatic invasion (LI) is defined as $\mathrm{S} 100$ protein-positive cells within a podoplanin-positive lymphatic space. This dual immunohistochemical staining should be performed in all cases of MELTUMP, because it can provide a prognostic adjunct in determining whether those lesions are capable of distant metastases and fatal outcomes (2). The presence of LI correlates with a more aggressive clinical outcome, defined as developing nodal metastases, distant metastases, or melanoma-related death (2). Various studies have demonstrated the utility of FISH as an ancillary method in the diagnosis of ambiguous melanocytic neoplasms. A panel of FISH targeting loci on chromosomes 6 and 11 has emerged as a powerful tool to discriminate melanoma from nevi with a sensitivity and specificity of $87 \%$ and $96 \%$, respectively (3). Early retrospective studies have shown correlation between metastatic behavior among ambiguous melanocytic lesions and FISH results (4). FISH testing can so help to reduce the number of equivocal diagnoses in course of ambiguous melanocytic neoplasm, in particular if FISH testing is positive. In the study of Cerroni et al. (1), 75 cases of MELTUMP were classified within three groups according to their behavior as follows: a) favorable (no evidence of metastatic disease after a follow-up $\geq 5$ years); b) unfavorable (tumor-related death and/or large deposits in the lymphatic nodes and/or visceral metastases; c) borderline (small nodal deposits of tumor cells $\leq 0.2 \mathrm{~mm}$ ). The only three histopathologic criteria that were statistically different between the groups of unfavorable and favorable cases were: presence of mitoses, mitoses near to the base and an inflammatory reaction. All these features were found more frequently in cases with unfavorable behavior. In our experience (5), the second opinion of Prof. Elder and Prof. Murphy has permitted a better comprehension of MELTUMP. We have read with great interest the clinicopathological study of atypia and differential diagnosis in cellular blue nevi (CBN) by Yaman et al. (6), based on a 21-case series diagnosed between 2000-2014. Five patients were assessed as 'atypical cellular blue nevus' (ACBN). A 6-cm-diameter tumor showed an infiltrative development; two cases presented two mitoses and two MIB-1 labeling indexes at $3 \%$ and $2 \%$, respectively; a case was characterized by one mitosis and a confluent development, and a case by one mitosis with additional focal necrosis. No lymphatic and/or distant metastases were observed during the followup. Yaman et al. believe that there is no precise definition of ACBN, but the term is used for CBN that has atypical features and requires differentiation from malignant blue nevus. The features of ACBN are widely studied in the accurate review of blue nevi and variants by Zembowicz and Phadke (7). The authors believe that there are no established consensus criteria regarding diagnostic category of ACBN. This term is useful to identify histologically ambiguous lesion and to convey out some uncertainty in relation to the biological potential of the lesion, without overinterpreting the tumor as an outright melanoma. Zembowicz and Mihm (8) consider CBN as atypical when they are large $(>5-10 \mathrm{~cm})$ and ulcerated, they show marked nuclear pleomorphism, and have more than 3 to 4 mitotic figures / square $\mathrm{mm}$, and either pushing or infiltrating margins. The authors argue that unfortunately these features are not discriminating and can also be found in conventional CBN, as well in malignant blue nevus. In the opinion of Murali et al. (9), the pathological features of ACBN are as follows: size greater than $3 \mathrm{~cm}$, increased cellularity, cellular polymorphism (focal areas of atypia in a background of CBN), increased mitotic activity (but less than 2 mitoses / square mm), no atypical mitoses and no areas of necrosis. In conclusion, ACBN is a classic example of MELTUMP, because divergent 
opinions regarding histological features and clinical behavior are present. The diagnosis of MELTUMP, referred to as an atypical blue nevus, seems to be more appropriate for determining an accurate management of the lesion.

\section{REFERENCES}

1. Cerroni L, Barnhill R, Elder D, Gottlieb G, Heenan P, Kutzner $\mathrm{H}$, LeBoit PE, Mihm M Jr, Rosai J, Kerl H. Melanocytic tumors of uncertain malignant potential: Results of a tutorial held at the XXIX Symposium of the International Society of Dermatopathology in Graz, October 2008. Am J Surg Pathol. 2010;34:314-26.

2. Abraham RM, Karakousis G, Acs G, Ziober AF, Cerroni L, Mihm $\mathrm{MC}$ Jr, Elder DE, Xu X. Lymphatic invasion predicts aggressive behavior in melanocytic tumors of uncertain malignant potential (MELTUMP). Am J Surg Pathol. 2013;37:669-75.

3. North JP, Garrido MC, Kolaitis NA, LeBoit PE, McCalmont TH, Bastian BC. Fluorescence in situ hybridization as an ancillary tool in the diagnosis of ambiguous melanocytic neoplasms: A review of 804 cases. Am J Surg Pathol. 2014;38:824-31.

4. Vergier B, Prochazkova-Carlotti M, de la Fouchardière A, Cerroni L, Massi D, De Giorgi V, Bailly C, Wesselmann U, Karlseladze A, Avril MF, Jouary T, Merlio JP. Fluorescence in situ hybridization, a diagnostic aid in ambiguous melanocytic tumors: European study of 113 cases. Mod Pathol. 2011;24:613-23.

5. Pusiol T, Morichetti D, Piscioli F, Zorzi MG. Theory and practical application of superficial atypical melanocytic proliferations of uncertain significance (SAMPUS) and melanocytic tumours of uncertain malignant potential (MELTUMP) terminology: Experience with second opinion consultation. Pathologica. 2012;104:70-7.
6. Yaman B, Kandiloglu G, Kumbaraci BS, Akalin T. Atypia and differential diagnosis in cellular blue nevi: Clinicopathological study of 21 cases. Turk Patoloji Derg. 2015;31:89-94.

7. Zembowicz A, Phadke PA. Blue nevi and variants: An update. Arch Pathol Lab Med. 2011;135:327-36.

8. Zembowicz A, Mihm MC. Dermal dendritic melanocytic proliferations: An update. Histopathology. 2004;45:433-51.

9. Murali R, McCarthy SW, Scolyer RA. Blue nevi and related lesions: A review highlighting atypical and newly described variants, distinguishing features and diagnostic pitfalls. Adv Anat Pathol. 2009;16:365-82.

\section{Francesco PISCIOLI}

Provincial Health Care Services, Institute of Pathology, Santa Maria del Carmine Hospital, ROVERETO (TN), ITALY

Teresa PUSIOL

Provincial Health Care Services, Institute of Pathology, Santa Maria del Carmine Hospital, ROVERETO (TN), ITALY

\section{Luca RONCATI}

Provincial Health Care Services, Institute of Pathology, Santa Maria del Carmine Hospital, ROVERETO (TN), ITALY

Department of Diagnostic and Clinical Medicine and of Public Health, Section of Pathology, University of Modena and Reggio Emilia, MODENA (MO), ITALY

E-mail: emailmedical@gmail.com Phone: +390594224812 